Check for updates

Cite this: RSC Adv., 2017, 7, 18046

\title{
Mechanically strong interpenetrating network hydrogels for differential cellular adhesion
}

Received 30th January 2017 Accepted 6th March 2017

DOI: $10.1039 / \mathrm{c} 7 \mathrm{ra01271c}$

rsc.li/rsc-advances

\begin{abstract}
Chong Shen, Yuyan Li, Huadi Wang and Qin Meng (D)*
Hydrogels as "soft-and-wet" materials have been widely used as tissue engineering scaffolds due to their similarity to natural extracellular matrix. However, it remains extremely challenging to develop mechanically strong hydrogels that can stimulate desirable mammalian cell adhesion but reduce the probable fouling from microbes and other unwanted cells. To achieve this purpose, we fabricated interpenetrating network (IPN) hydrogels consisting of cell-adhesive gelatin and non-fouling carboxybetaine (CBMA) via a "one-pot" synthesis process. Far stronger than their parent gels of gelatin and PCBMA, the IPN gels presented compressive and stretch fracture stresses over 6.5 and $2.4 \mathrm{MPa}$, and failure strains over $95 \%$ and $700 \%$, respectively. The obtained IPN gels only allowed the adhesion and confluence of parenchymal mammalian cells (e.g. human umbilical vein endothelial cells, HUVEC; smooth muscle cells, SMC) but resisted well the attachment of platelets and microbes. In this regard, the CBMA/gelatin IPN gels can be potentially used in the construction of artificial soft tissues such as blood vessels because of their specific mechanical and differential adhesive properties.
\end{abstract}

\section{Introduction}

Most native tissues (e.g. muscle and blood vessel) in the human body have high water contents (30-80\%) but present excellent mechanical performances. ${ }^{1}$ Organized by the cell population and extracellular matrix, native tissues are able to regulate the parenchymal cell behaviors (proliferation, migration, functional expression, etc.), while acting as natural barriers to the invasion/adhesion of foreign cells (e.g. microbes and platelets). ${ }^{2}$ To mimic the real tissues, it has been a challenge to seek soft but mechanically strong materials that can stimulate desirable cell adhesion but reduce the fouling of unwanted cells. Such materials are expected to simultaneously promote tissue interactions and suppress implant-associated infection in clinical practice. $^{3}$

Hydrogels, consisting of cross-linked macromolecules and water, are soft and wet materials mimicking soft tissues well. ${ }^{4}$ But most conventional hydrogels are weak and brittle, ${ }^{1,5}$ with a failure tensile stress far lower than those of native soft tissues. Accordingly, many efforts have been made to synthesize tough hydrogels such as slide-ring gels, ${ }^{6}$ tetra-PEG gels, ${ }^{7}$ nanocomposite gels ${ }^{8}$ and double-network gels. ${ }^{9}$ Despite the success on improving the mechanical property, the fabrication of these hydrogels often involves cytotoxic chemicals, limiting their applications as cell-contacted materials. ${ }^{\mathbf{1 0}}$ For example, polyacrylamide, the most used chemicals in double-network gels, ${ }^{\mathbf{1 , 5}}$

College of Chemical and Biological Engineering, Zhejiang University, 38 Zheda Road, Hangzhou, Zhejiang 310027, PR China. E-mail: mengq@zju.edu.cn is highly toxic during degradation, while acrylamido-free gels were either mechanically weak ${ }^{11}$ or difficult for fabrication. ${ }^{12}$

As known, "cell-compatible" hydrogels actually include two categories: one contains cell recognized sites and stimulates cell adhesion/spreading/proliferation, such as protein gels of collagen, ${ }^{13}$ gelatin ${ }^{14}$ and fibrin; ${ }^{15}$ another is cell inert gels lacking biological moieties, such as zwitterionic polymers and polyethylene glycol gels. ${ }^{16}$ But the cell adhesive gels also enabled the adhesion of microbes and other unwanted cells (e.g. platelets) ${ }^{17}$ while the cell inert gels indiscriminately resisted all cell adhesion. In this regard, the hydrogels that can stimulate desirable mammalian cell adhesion while reduce the probable fouling of microbes and other unwanted cells are still lacked.

To obtain the hydrogels with differential adhesive surfaces to various cells, cell-adhesive gelatin and non-fouling carboxybetaine (CBMA) are blended to fabricate the interpenetrating network (IPN) hydrogels in this study. By tuning the gelatin/ CBMA ratios, high mechanical property and differential adhesive surface will be achieved on the same gel. Such IPN gels would be superior to conventional hydrogels and hard materials in construction of artificial soft tissues on both promoting tissue healing and reducing the risk of infection.

\section{Materials and methods}

\section{Hydrogel preparation}

2-Carboxy- $N, N$-dimethyl- $N$-(2'-(methacryloyloxy)ethyl)ethanaminium inner salt (carboxybetaine methacrylate, CBMA) was synthesized according to the previously reported method. ${ }^{18}$ 
CBMA/gelatin IPN gel cylinders were prepared by one-pot method with mixture of CBMA and gelatin (Sigma-Aldrich, St. Louis, MO) solution in 24-well plate, while pure pCBMA and gelatin gels were prepared as controls. Briefly, gelatin was dissolved into hot water at concentration of $20 \% \mathrm{w} / \mathrm{v}$ as solution $\mathrm{A}$. The CBMA (0.2-1 M), PEGDA (0.02-0.1 M, Sigma-Aldrich), genipin (2 mg mL $\mathrm{mL}^{-1}$, Sigma-Aldrich) and Irgacure $2959(8 \mathrm{mg}$ $\mathrm{mL}^{-1}$, BASF) were dissolved into water to prepare solution B. Then solution A and B were well mixed at ratio of $1: 1$. All the components in gel solutions were listed in Table 1.

By casting the gel solution into the cylindrical mould, it was placed under a crosslinker with UV intensity of $50 \mathrm{~mW} \mathrm{~cm}^{-2}$ for $1 \mathrm{~h}$. After photo polymerization under UV irradiation, the primary formed gels were kept in room temperature at least $24 \mathrm{~h}$ for genipin. Then the IPN gels were immersed in pure water for 1 week until they reached swelling equilibrium. In addition, the pure genipin gels were prepared without UV irradiation.

\section{Hydrogel characterization}

The mechanical properties of gels were measured by compressive testing using the Instron Series IX Automated Materials Testing System (Zwick/Roell Z020). ${ }^{19}$ Hydrogel cylinders with 15 $\mathrm{mm}$ in diameter and $20 \mathrm{~mm}$ in height were placed on the center of the lower compression plate at $37{ }^{\circ} \mathrm{C}$ with humidity of $50 \%$. The sample was then compressed by the upper plate, by connecting to a $500 \mathrm{~N}$ load cell, at a crosshead speed of $5 \mathrm{~mm}$ $\min ^{-1}$. The fracture stress and strain were determined as the nominal stress and strain at the failure point, respectively. Young's modulus was determined as the slope at the 0-0.1 strain range from the stress-strain curve. Measurements were performed six times for each sample.

Swelling ratio of gels was detected by cutting the gel cylinders $(15 \mathrm{~mm} \times 20 \mathrm{~mm})$ into $4-6$ of small pieces and placing them in distilled water at $25{ }^{\circ} \mathrm{C}$. The gels were gently shaken for $24 \mathrm{~h}$ and were measured to assess the hydrated weight $\left(W_{\mathrm{h}}\right)$. Then the hydrated gels were dried under nitrogen atmosphere at $100{ }^{\circ} \mathrm{C}$ for $24 \mathrm{~h}$. The weight was recorded as the dry weight $\left(W_{\mathrm{d}}\right)$ of the gel. The experiment was repeated five times to obtain average values. The swelling ratio was calculated as $W_{\mathrm{h}} / W_{\mathrm{d}}$.

To observe the porous morphology, the gel samples were frozen at $-80{ }^{\circ} \mathrm{C}$ and dried in vacuum in a freeze dryer. The dried gels were detected by scanning electron microscope (SEM, HITACHI TM-1000, Japan) after gold-palladium coating.

The hydration of gels was determined by thermogravimetric analysis (TGA) by heating the gels from room temperature to $500{ }^{\circ} \mathrm{C}$ at a heating rate of $5{ }^{\circ} \mathrm{C} \min ^{-1}$ under nitrogen flow. Gel samples after swelling equilibrium were taken out from pure water and samples ranging from 4 to $8 \mathrm{mg}$ in weight were tested in platinum pans. The weight loss of gels with the increased temperature was recorded by a thermogravimetric analyzer (Perkin-ElmerPyris-6, Wellesley, MA).

\section{Mammalian cell attachment and growth on hydrogel surfaces}

Human umbilical vein endothelial cells (HUVEC) and human aorta smooth muscle cells (SMC) were purchased from ATCC. HUVECs were maintained in culture with endothelial cell growth medium 2 (EGM-2, Lonza). SMCs were cultured in DMEM medium (high glucose, Gibco) supplemented with $10 \%$ FBS.

Both of the cells were seeded on the top of gel surfaces at density of $1 \times 10^{5}$ cells per $\mathrm{cm}^{2}$ for $4 \mathrm{~h}$. After attachment, the gels were carefully rinsed with phosphate buffered saline (PBS) to remove unattached cells. The gels were then immersed in trypsin-EDTA $(0.25 \%$ vs. $0.02 \%)$ solution to lift the adhered cells. After incubation for $20 \mathrm{~min}$, the trypsin-EDTA solution was neutralized with culture medium. The cell numbers on gels were counted for triplicate samples using a hemacytometer. The percent of attached cells was calculated as follows:

$$
\% \text { attachment }=\text { cells } \mathrm{s}_{\text {on hydrogel }} / \text { cells }_{\text {seeded }} \times 100 \%
$$

At 1, 3, 5 and 7 days culture, the proliferation of cells on gel surfaces were detected by MTT reduction according to previously reported method. ${ }^{20}$ After 7 days culture, the cells were fixed with $4 \%$ paraformaldehyde for further SEM and confocal observation. For immunostaining of HUVEC and SMC, the VEcadherin and $\alpha$-SMA was stained by mouse monoclonal primary antibody (Abcom) and Alexa Flour 488 goat-anti-mouse secondary antibody (Invitrogen). Nuclear staining was performed by mounting medium containing DAPI (Vector Laboratories). Confocal microscopy (Carl Zeiss LSM 5 Exciter) was used to visualize and capture cells with good resolution.

\section{Adhesion of bacteria and platelets on hydrogels}

To detect the bacteria adhesion, Pseudomonas aeruginosa in PBS at $5 \times 10^{7} \mathrm{~mL}^{-1}$ were seeded on the top surface of hydrogels. After $1 \mathrm{~h}$ of incubation at $37^{\circ} \mathrm{C}$, the gels were rinsed with PBS and soaked into Tryptic Soy Broth (TSB) for incubation of 1, 2, 3 and $4 \mathrm{~h}$ at $37^{\circ} \mathrm{C}$. At each time point, the gels were rinsed by PBS, fixed using $4 \%$ paraformaldehyde and stained by DAPI before observation under fluorescence microscope (OLYMPUS Ix70). The cell density was analyzed by counting the number of bright dots in randomly selected area using Image J. ${ }^{21}$ For detecting platelet adhesion, the platelet-rich plasma was separated from fresh human blood by centrifugation and diluted to $2-5 \times 10^{8}$ platelet per $\mathrm{mL}$. The study was performed in accordance with guidelines and regulations of Zhejiang University (Zhejiang, China) and approved by the Ethical and Research Committee of Zhejiang University. Various gels were incubated in platelet suspension at $37{ }^{\circ} \mathrm{C}$ for $1 \mathrm{~h}$, then rinsed with warm PBS, and stained by calcein-AM at $1 \mu \mathrm{M}$. The platelet density on gels was quantified by assessing the release of $\mathrm{LDH}$ in the Triton-X lysing solution with a LDH assay kit (Saike Bio. Co. Ningbo, China).

\section{Statistical analysis}

All data from cell experiments were analyzed by means \pm SD from three independent experiments. Comparisons between multiple groups were performed with the ANOVA test by SPSS, or results from two different groups were tested with the unpaired Student $t$-test. $P$-Values less than 0.05 were considered statistically significant. 


\section{Results}

\section{Higher mechanical property of CBMA/gelatin IPN gels than their parent gelatin and pCBMA gels}

Gel cylinders with $15 \mathrm{~mm}$ in diameter and $20 \mathrm{~mm}$ in height were prepared according to the compositions in Table 1 for comparing their mechanical properties. As expected, CBMA/ gelatin IPN gels (1-9\#) showed 4-20 folds higher fracture stress than either of their parents (i.e., pure gelatin and PCBMA gels, Table 2), while increased CBMA and crosslinker (polyethylene oxide diacrylate, PEGDA, MW 550) concentrations improved the hardness but exacerbated the brittleness of IPN gels. Especially, 1-3\# IPN gels have fracture stress of >6.5 MPa, compressive fracture strain about $90 \%$ and modulus $>100 \mathrm{kPa}$ (Table 2).

As shown in Fig. 1, the CBMA/gelatin IPN gels turned to present dark blue due to genipin crosslinking. The IPN-1 gel could withstand high-level deformations of bending (Fig. 1A)

Table 1 Components for hydrogel fabrication

\begin{tabular}{llllll}
\hline & & & & & \\
No. & $\begin{array}{l}\text { Gelatin } \\
(\mathrm{w} / \mathrm{v} \%)\end{array}$ & $\begin{array}{l}\text { CBMA } \\
(\mathrm{M})\end{array}$ & $\begin{array}{l}\text { PEGDA } \\
(\mathrm{M})\end{array}$ & $\begin{array}{l}\text { Genipin } \\
\left.(\mathrm{mg} \mathrm{mL})^{-1}\right)\end{array}$ & $\begin{array}{l}2959 \\
\left(\mathrm{mg} \mathrm{mL}^{-1}\right)\end{array}$ \\
\hline IPN-1 & 10 & 0.1 & 0.01 & 1 & 4 \\
IPN-2 & 10 & 0.2 & 0.01 & 1 & 4 \\
IPN-3 & 10 & 0.5 & 0.01 & 1 & 4 \\
IPN-4 & 10 & 0.1 & 0.025 & 1 & 4 \\
IPN-5 & 10 & 0.2 & 0.025 & 1 & 4 \\
IPN-6 & 10 & 0.5 & 0.025 & 1 & 4 \\
IPN-7 & 10 & 0.1 & 0.05 & 1 & 4 \\
IPN-8 & 10 & 0.2 & 0.05 & 1 & 4 \\
IPN-9 & 10 & 0.5 & 0.05 & 1 & 4 \\
pCBMA- & 0 & 0.5 & 0.01 & 0 & 4 \\
1 & & & & & 4 \\
pCBMA- & 0 & 0.5 & 0.025 & 0 & 4 \\
2 & & & & & \\
pCBMA- & 0 & 0.5 & 0.05 & 0 & \\
3 & & & & &
\end{tabular}

and knotting (Fig. 1B) without any observable damage. Besides, the gel could be stretched for at least 4 times (Fig. 1C). Particularly, the IPN-1 gel quickly recovered to the initial shape after removal of the deformation force (Fig. 1D), indicating the shape-recovery property. The mechanical property of 1-3\# IPN gels was further quantified by the strain-stress curves (Fig. 2A). IPN-1 and IPN-2 gels ruptured at stress of $>2.4 \mathrm{MPa}$ and strain of $>700 \%$, while IPN-3 gel was weaker and more brittle. Their parent gels have not been involved in Fig. 2A because they ruptured at an undetectable low level (data not shown).

As reflected by the TGA data (Fig. 2B), IPN gels showed the slower dehydrated rate than the pure gelatin gel, due to the higher capacity of pCMBA network on binding water. The CBMA/gelatin IPN gels exhibited the porous surfaces with pore size ranging from 30 to $60 \mu \mathrm{m}$ under SEM observation (Fig. 2C-E), similar with previously reported double-network hydrogels. ${ }^{22}$

\section{Mammalian cells well adhere and proliferate on surface of CBMA/gelatin IPN gels}

As the physiological interactions with cells were a critical property for tissue-contacted biomaterials, ${ }^{23}$ the effects of various hydrogels on stimulating desirable mammalian cell adhesion and proliferation were then detected.

As shown in Fig. 2A, the gelatin gel was the best surface for adhesion of HUVEC and SMC, while pCBMA gel could not allow the cell adhesion at all. The adhesive ratio on IPN-1 and IPN-2 gels was still over $50 \%$, but that on IPN-3 gel with increased CBMA content was less than $30 \%$ (Fig. 3A). The lower cell attachment on IPN gels led to the slower cell growth than that on gelatin gel, but the cell proliferation on IPN-1 and IPN-2 gels reached to the same level as that on gelatin gel after 7 days culture (Fig. 3B and C). By contrast, the cell population on IPN-3 and pCBMA gels didn't increase with culture time, indicating the halted proliferation of cells (Fig. 3B and C).

As confirmation, Fig. 4 indicated that the HUVEC expressed VE-cadherin (stained green, right images in Fig. 4A and C) and covered the surface of IPN-1 and IPN-2 gels (SEM image, left in Fig. 4A and C) at 7 days culture after seeding. The SMC similarly

Table 2 Mechanical property of various hydrogels

\begin{tabular}{|c|c|c|c|c|}
\hline IPN-1 & $6.58 \pm 3.51$ & $95.4 \pm 8.5$ & $109.1 \pm 10.6$ & $8.0 \pm 0.1$ \\
\hline IPN-3 & $10.27 \pm 3.96$ & $85.1 \pm 5.5$ & $158.8 \pm 23.1$ & $6.2 \pm 0.2$ \\
\hline IPN-4 & $3.55 \pm 0.81$ & $85.5 \pm 10.5$ & $122.9 \pm 5.9$ & $7.4 \pm 0.3$ \\
\hline IPN-5 & $5.24 \pm 1.20$ & $70.1 \pm 15.9$ & $125.6 \pm 22.8$ & $6.5 \pm 0.2$ \\
\hline IPN-8 & $3.68 \pm 0.96$ & $51.4 \pm 9.6$ & $144.1 \pm 24.8$ & $4.5 \pm 0.2$ \\
\hline IPN-9 & $4.35 \pm 1.01$ & $45.2 \pm 10.4$ & $198.5 \pm 21.4$ & $3.2 \pm 0.4$ \\
\hline pCBMA-1 & $0.54 \pm 0.27$ & $56.9 \pm 11.5$ & $9.2 \pm 1.5$ & $11.2 \pm 0.5$ \\
\hline pCBMA-2 & $0.75 \pm 0.18$ & $49.4 \pm 10.5$ & $16.1 \pm 5.0$ & $7.8 \pm 0.1$ \\
\hline pCBMA-3 & $0.74 \pm 0.12$ & $18.7 \pm 3.9$ & $33.2 \pm 16.3$ & $3.5 \pm 0.4$ \\
\hline Gelatin & $0.65 \pm 0.12$ & $72.5 \pm 10.4$ & $78.7 \pm 10.2$ & $8.5 \pm 0.2$ \\
\hline
\end{tabular}


A

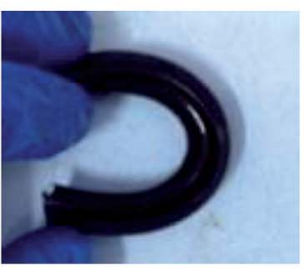

B

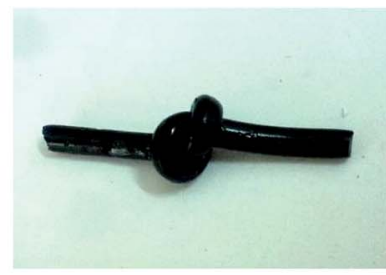

C

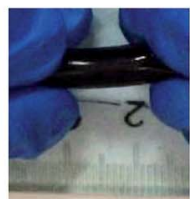

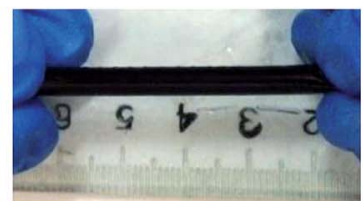

D

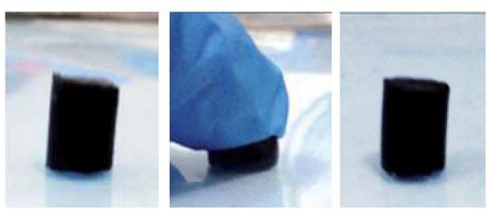

Fig. 1 CBMA/gelatin IPN gels show extraordinary mechanical properties: (A) bending; (B) knotting; (C) stretching and (D) compression.
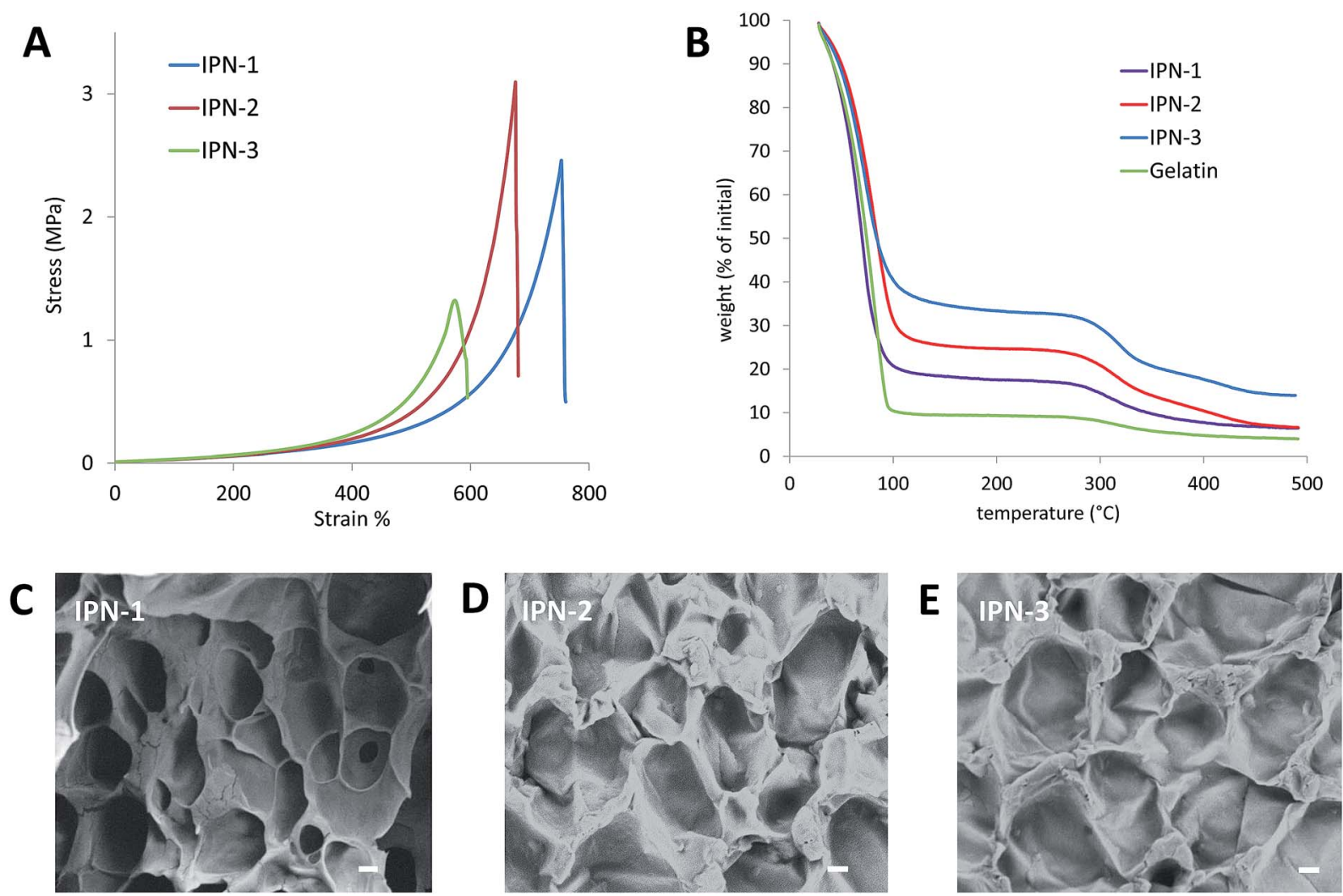

Fig. 2 (A) Tensile stress-strain behaviors of IPN gels prepared at various CBMA concentrations. (B) Weight loss of hydrogels with the increased temperature detected by thermogravimetric analysis (TGA). (C-E) Surface of CBMA/gelatin IPN gels under scanning electron microscopy observation. Scale bar $=10 \mu \mathrm{m}$.

formed confluent cell layer on surface of IPN-1 and IPN-2 gels with expression of $\alpha$-SMA (stained green, Fig. 4B and D).

\section{CBMA/gelatin IPN gels display anti-adhesive property to bacteria and platelets}

In IPN gels, CBMA was the anti-fouling composition, but gelatin could facilitate the cell adhesion. In this regard, the anti- adhesive effects of IPN-1 and IPN-2 gels were evaluated by the attachment test of platelets and bacteria of $P$. aeruginosa.

As expected, the presence of CBMA in IPN gels greatly reduced the adhesive capability to platelets (stained green by calcein-AM, Fig. 5A), while IPN-2 gel better resisted the platelets due to its higher CBMA content (Fig. 5A and B). Similarly, the $P$. aeruginosa stained by DAPI largely attached on surface of gelatin gel but only scattered deposited on IPN gels after $1 \mathrm{~h}$ of 

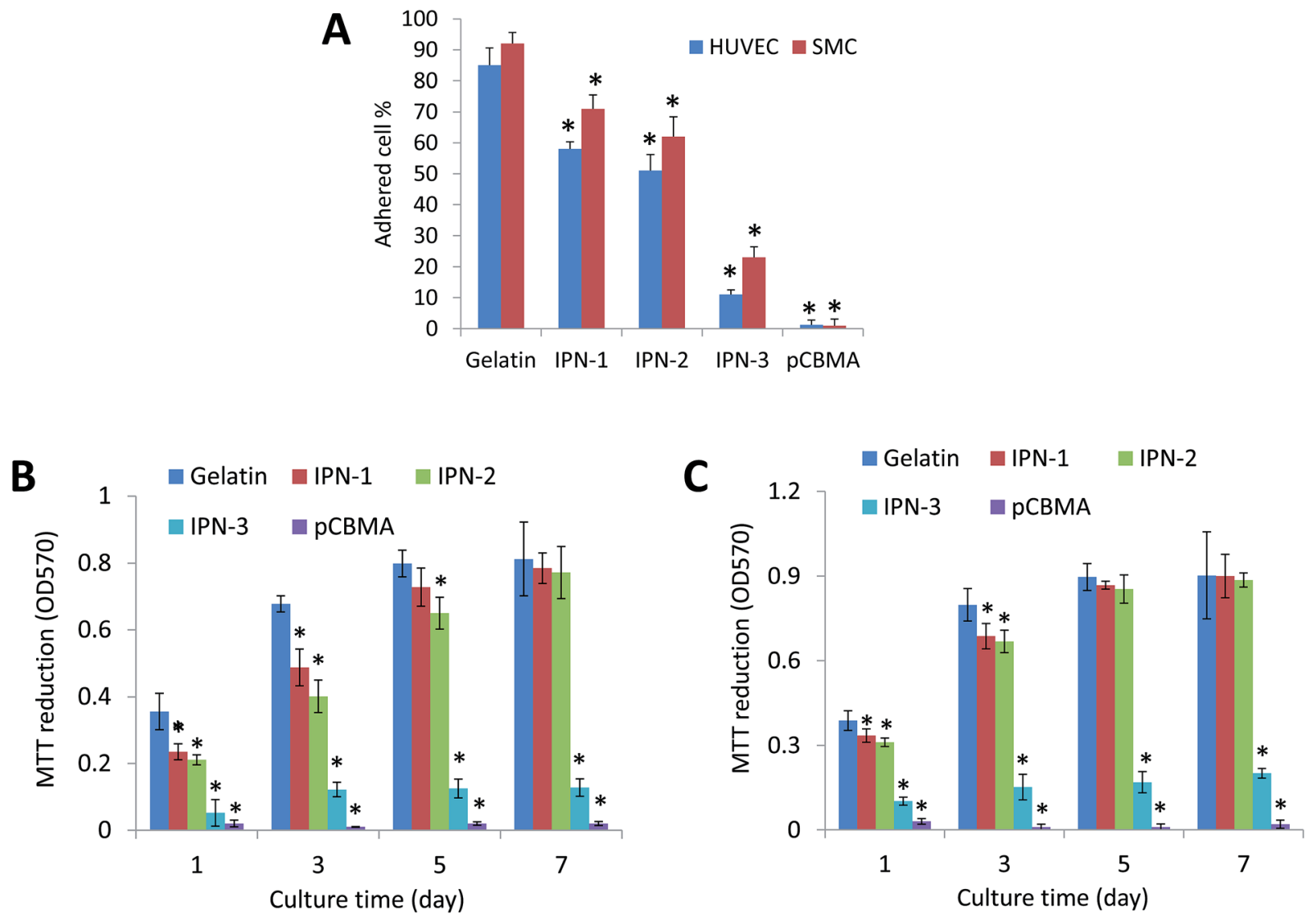

Fig. 3 Human umbilical vein endothelial cells (HUVEC) and smooth muscle cells (SMC) attachment and growth on surfaces of various hydrogels. (A) Adhesive ratio of cells on hydrogels after 4 h of attachment. ${ }^{*} p<0.05$. HUVEC (B) and SMC (C) growth on hydrogels during 1-7 days culture as indicated by MTT reduction. ${ }^{*} p<0.05$.

HUVEC, IPN-1

A

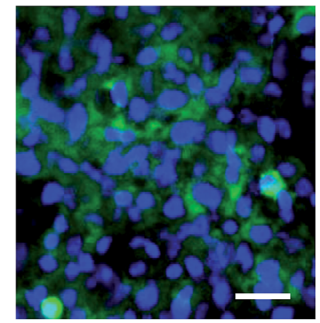

HUVEC, IPN-2

C

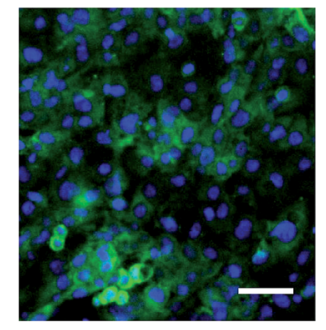

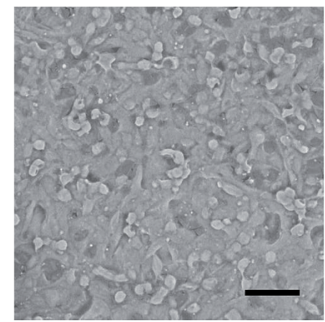

B
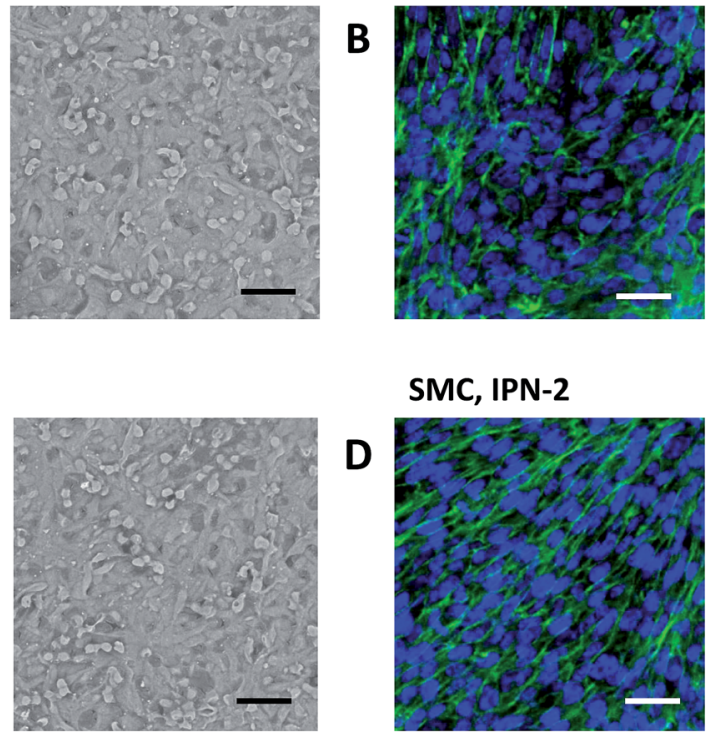

SMC, IPN-2

SMC, IPN-1

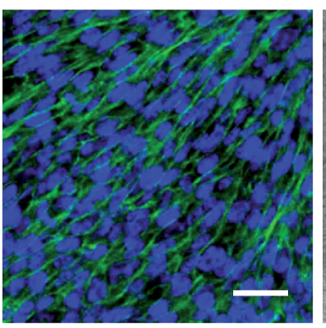

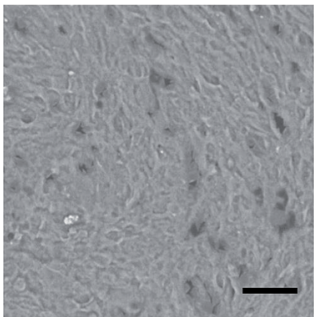

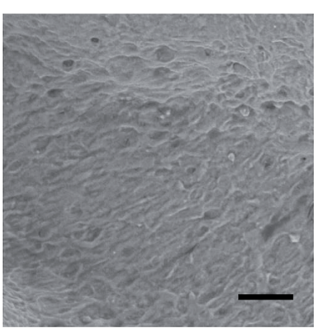

Fig. 4 (A and C) Morphology of human umbilical vein endothelial cells (HUVEC) on IPN hydrogels after 7 days culture. Left: Immunofluorescence staining of VE-cadherin (green). DAPI was used for nuclear counterstaining (blue). Right: Scanning electron microscopy images. (B and D) Morphology of smooth muscle cells (SMC) on IPN hydrogels after 7 days culture. Left: Immunofluorescence staining of $\alpha$-SMA (green). DAPI was used for nuclear counterstaining (blue). Right: Scanning electron microscopy images. Scale bars $=20 \mu \mathrm{m}$. 

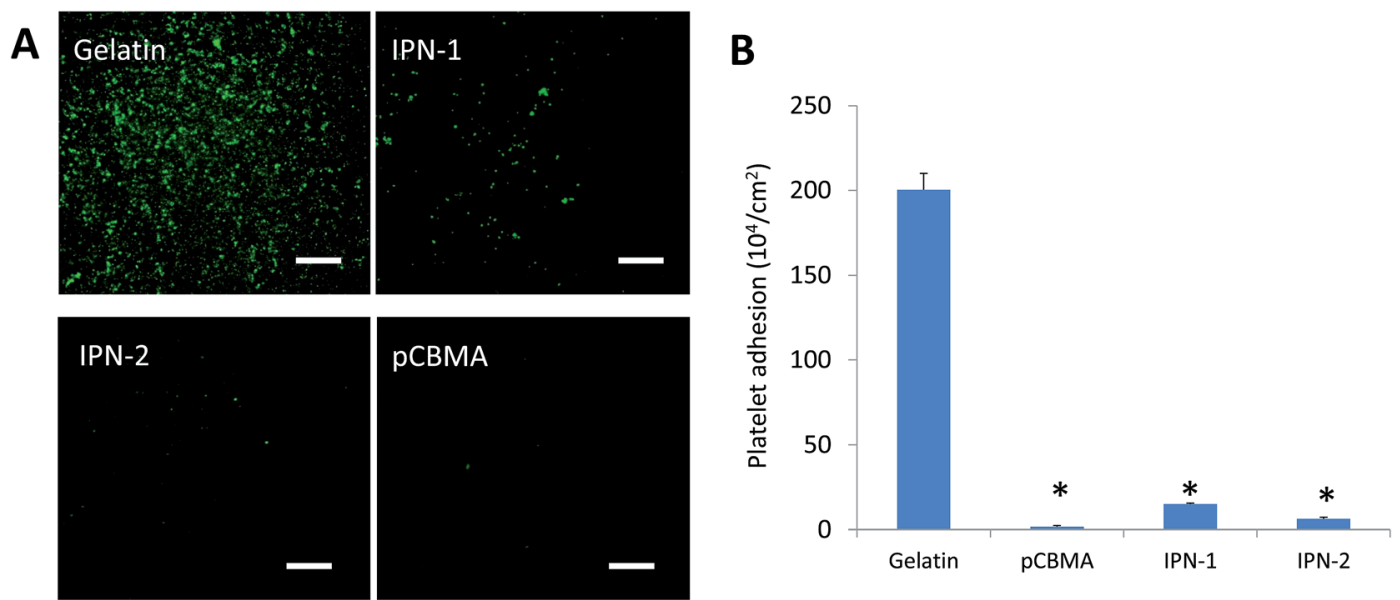

Fig. 5 Adhesion of platelets on various hydrogels. (A) Adhered platelets stained green by calcein. Scale bar $=10 \mu \mathrm{m}$. (B) Platelet adhesion detected by LDH leakage. ${ }^{*} p<0.05$.
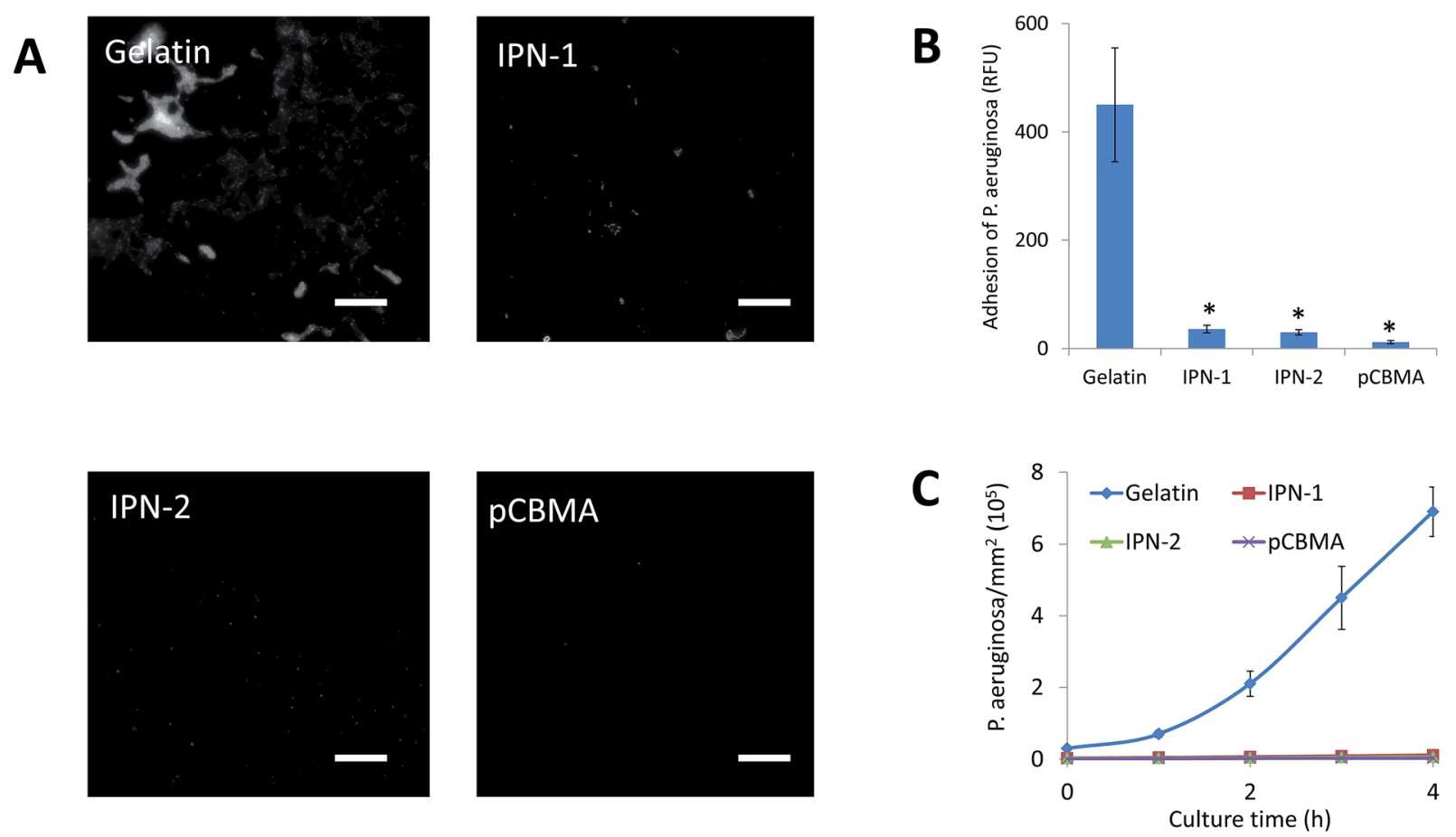

Fig. 6 Adhesion of $P$. aeruginosa on the various hydrogels. (A) Adhered $P$. aeruginosa stained by DAPI (bright dots). Scale bar $=5 \mu$ m. (B) Adhesion of $P$. aeruginosa on hydrogels as indicated by relative fluorescence unit of DAPI. ${ }^{*} p<0.05$ (C) $P$. aeruginosa growth within 4 h of incubation in Tryptic Soy Broth medium on the various hydrogels.

incubation with bacteria in PBS (Fig. 6A). The subsequent growth of bacteria on gel surfaces was also suppressed by the presence of CBMA, while the number of bacteria on gelatin gel increased almost linearly with incubation time (Fig. 6B).

\section{Discussion}

In IPN gels, polymerized CBMA performed as the rigid first network via quick and high crosslinking of PEGDA within $1 \mathrm{~h}$ before the slow reaction of genipin with gelatin during $24 \mathrm{~h}$. As pCBMA is not a typical polyelectrolyte at physical $\mathrm{pH}$, its hydrogels presented lower swelling ratios (3.5-11.2, Table 2) than the typical polyelectrolyte gels (e.g. 175 for poly(2-acrylamido-2-methylpropanesulfonic) ${ }^{19}$ ), which benefited to the maintenance of the IPN gel shapes and mechanical property during swelling. The genipin crosslinked gelatin acted as the second network via the ductile connections among gelatin chains. ${ }^{24}$ The flexibility of genipin-gelatin chains enhanced the fracture strain of IPN gels to more than $700 \%$ (Fig. 2A), while the same IPN gels crosslinked by carbodiimide, a zero-length crosslinker, were much more brittle (data not shown). By comparison, the CBMA/gelatin IPN gels, though still weaker 
than acrylamide-containing double-network gels (compressive fracture stress of $20-60 \mathrm{MPa}$, strain of $90-95 \%),{ }^{1}$ was stronger than other double-network zwitterionic gels (failure stress < $0.6 \mathrm{MPa}$, failure strain $<60 \%)^{25}$ and acrylamide-free IPN gels (e.g. PEG/agarose hydrogel with failure stress $<1.6 \mathrm{MPa}$ ). ${ }^{22}$ Having avoided the highly toxic polyacrylamide, the CBMA/ gelatin IPN gels with high mechanical property would be superior to current double-network hydrogels in tissue engineering applications.

Fabrication of surfaces that allow the desirable cell attachment but prevent the unwanted fouling is a challengeable and critical problem in tissue engineering, since normal surfaces do not show differential adhesive effects to different cells. To our knowledge, the only reported surfaces for differential adhesion to mammalian cells and microbes were the PEG patterned or microgel modified surfaces with specific inter-gel spacings. ${ }^{17,21}$ But their fabrication was complex and did not feasible for modification of soft materials (e.g. hydrogels). By contrast, we obtained the differential adhesive surfaces via simply tuning the contents of non-adhesive and adhesive components (i.e. CBMA and gelatin) in the hydrogels. This may provide a robust way to engineer the differential adhesive surfaces for reducing the implant-associated infection and blood-contact fouling. ${ }^{3}$

The mechanism on the differential adhesive effects of IPN gels might be related to the different sizes of parenchymal mammalian cells $(10-20 \mu \mathrm{m})$, platelets $(1-2 \mu \mathrm{m})$ and bacteria (about $1 \mu \mathrm{m}$ ). Similar to the surfaces with defined PEG patterns at inter-gel spacing of 1-2 $\mu \mathrm{m}$ that allowed the adhesion of mammalian cells but resisted the bacteria, ${ }^{3,17}$ the pCBMA on IPN gel surfaces might formed dense non-fouling domains at comparable spacing to platelets/bacteria but far small than HUVEC/SMC cells. As bacterial adhesion has been known to be mediated by maximizing the cell-substrate contact and minimizing the cell deformation from the thermodynamic view, ${ }^{26}$ some studies reported the resistance of bacterial adhesion by surface patterns with size around 1-3 $\mu \mathrm{m}^{26,27}$ By contrast, parenchymal mammalian cells might be insensitive to small amount of non-adhesive molecules on surfaces due to the soft and flexible focal contacts. ${ }^{28}$ This could be supported by the results in our previous study ${ }^{29}$ and other literature. ${ }^{30}$ In this regard, bacteria and platelets might be hard to access to the adhesive sites on surface via sensing the non-adhesive features at spacing comparable to their own sizes. By contrast, parenchymal mammalian cells with modulated adhesiveness ${ }^{17}$ are able to adhere to the adhesive sites among non-adhesive domains by sub-micrometer focal contacts. ${ }^{17,21}$

Compared with native soft tissues, the IPN gels may most approach to the mechanical property and cell responsibility of blood vessels. ${ }^{31}$ Current engineered vessel grafts (e.g. electrospinning fibers, ${ }^{32}$ Teflon and Dacron ${ }^{33}$ ) are hard materials lacking elasticity. The mismatched the compliance of soft blood vessels elicited the intimal hyperplasia, thrombogenicity and turbulence in blood flow at the anastomotic site..$^{34}$ By contrast, the IPN gels were soft and elastic as native artery, ${ }^{31}$ which is possibly able to reduce the compliance mismatch in clinical use. Moreover, the IPN gels also present their advantages on friendly to endothelial cells and non-fouling for platelets, while synthetic scaffolds usually induced thrombus formation and could not promote the endothelial recellularization..$^{35}$ Nevertheless, the feasibility of CBMA/gelatin IPN gels as vessel grafts still need further investigation by in vitro experiments on burst pressure/compliance, and long-term animal testing, etc.

\section{Conclusions}

We fabricated the mechanically strong IPN hydrogels using celladhesive gelatin and non-fouling carboxybetaine (CBMA) by a "one-pot" synthesis process. By tuning the ratio of gelatin to CBMA, the obtained IPN gels presented a failure stress at $>2.4 \mathrm{MPa}$ and strain of $>700 \%$. The CBMA/gelatin IPN gels only allowed the adhesion and confluence of mammalian cells (HUVEC and SMC) but well resisted the fouling of platelets and microbes. The CBMA/gelatin IPN gels can be potentially used in construction of artificial soft tissues such as blood vessels because of their effects on promoting cell-substrate interaction and reducing the blood-contacted fouling or microbe infection.

\section{Acknowledgements}

We gratefully acknowledge the financial support of this study by NSFC (National Natural Science Foundation of China, No. 21176216 and 21276227$)$.

\section{References}

1 J. P. Gong, Soft Matter, 2010, 6, 2583.

2 L. G. Griffith and M. A. Swartz, Nat. Rev. Mol. Cell Biol., 2006, 7, 211.

3 Y. Wang, G. Subbiahdoss, J. Swartjes, H. C. van der Mei, H. J. Busscher and M. Libera, Adv. Funct. Mater., 2011, 21, 3916.

4 N. Annabi, A. Tamayol, J. A. Uquillas, M. Akbari, L. E. Bertassoni, C. Cha, G. Camci-Unal, M. R. Dokmeci, N. A. Peppas and A. Khademhosseini, Adv. Mater., 2014, 26, 85.

5 J. Y. Sun, X. Zhao, W. R. Illeperuma, O. Chaudhuri, K. H. Oh, D. J. Mooney, J. J. Vlassak and Z. Suo, Nature, 2012, 489, 133. 6 Y. Okumura and K. Ito, Adv. Mater., 2001, 13, 485.

7 T. Sakai, T. Matsunaga, Y. Yamamoto, C. Ito, R. Yoshida, S. Suzuki, N. Sasaki, M. Shibayama and U. I. Chung, Macromolecules, 2008, 41, 5379.

8 K. Haraguchi and T. Takehisa, Adv. Mater., 2002, 14, 1120.

9 J. Y. Sun, X. H. Zhao, W. R. K. Illeperuma, O. Chaudhuri, K. H. Oh, D. J. Mooney, J. J. Vlassak and Z. G. Suo, Nature, 2012, 489, 133.

10 Y. Zhao, T. Nakajima, J. J. Yang, T. Kurokawa, J. Liu, J. Lu, S. Mizumoto, K. Sugahara, N. Kitamura, K. Yasuda, A. U. Daniels and J. P. Gong, Adv. Mater., 2014, 26, 436.

11 (a) Y. Zhang, P. Heher, J. Hilborn, H. Redl and D. A. Ossipov, Acta Biomater., 2016, 38, 23-32; (b) S. M. Hong, D. Sycks, H. F. Chan, S. T. Lin, G. P. Lopez, F. Guilak, K. W. Leong and X. H. Zhao, Adv. Mater., 2015, 27, 4035.

12 A. Nakayama, A. Kakugo, J. P. Gong, Y. Osada, M. Takai, T. Erata and S. Kawano, Adv. Funct. Mater., 2004, 14, 1124. 
13 C. S. Szot, C. F. Buchanan, J. W. Freeman and M. N. Rylander, Tissue Eng., Part C, 2013, 19, 864.

14 M. Nikkhah, N. Eshak, P. Zorlutuna, N. Annabi, M. Castello, K. Kim, A. Dolatshahi-Pirouz, F. Edalat, H. Bae, Y. Yang and A. Khademhosseini, Biomaterials, 2012, 33, 9009.

15 S. K. Seidlits, C. T. Drinnan, R. R. Petersen, J. B. Shear, L. J. Suggs and C. E. Schmidt, Acta Biomater., 2011, 7, 2401.

16 Z. Zhang, T. Chao, L. Liu, G. Cheng, B. D. Ratner and S. Jiang, J. Biomater. Sci., Polym. Ed., 2009, 20, 1845.

17 Y. Wang, J. F. da Silva Domingues, G. Subbiahdoss, H. C. van der Mei, H. J. Busscher and M. Libera, Biomaterials, 2014, 35, 5446.

18 Z. Zhang, T. Chao, S. Chen and S. Jiang, Langmuir, 2006, 22, 10072.

19 H. Y. Yin, T. Akasaki, T. L. Sun, T. Nakajima, T. Kurokawa, T. Nonoyama, T. Taira, Y. Saruwatari and J. P. Gong, J. Mater. Chem. B, 2013, 1, 3685.

20 C. Shen, Q. Meng and G. Zhang, Biofabrication, 2015, 7, 015003.

21 Q. Wang, X. Yu and M. Libera, Adv. Healthcare Mater., 2013, 2, 687.

22 M. A. Haque, T. Kurokawa and J. P. Gong, Polymer, 2012, 53, 1805.

23 J. Thiele, Y. Ma, S. M. Bruekers, S. Ma and W. T. Huck, Adv. Mater., 2014, 26, 125.
24 H. G. Sundararaghavan, G. A. Monteiro, N. A. Lapin, Y. J. Chabal, J. R. Miksan and D. I. Shreiber, J. Biomed. Mater. Res., Part A, 2008, 87, 308.

25 Z. Zhang, T. Chao and S. Jiang, J. Phys. Chem. B, 2008, 112, 5327.

26 M. Yang, Y. Ding, X. Ge and Y. Leng, Colloids Surf., B, 2015, 135, 549.

27 A. I. Hochbaum and J. Aizenberg, Nano Lett., 2010, 10, 3717.

28 B. M. Gumbiner, Cell, 1996, 84, 345.

29 C. Shen, Q. Meng and G. Zhang, Biotechnol. Bioeng., 2013, 110, 2173.

30 C. H. Seo, K. Furukawa, K. Montagne, H. Jeong and T. Ushida, Biomaterials, 2011, 32, 9568.

31 (a) R. L. Armentano, D. B. Santana, E. I. Cabrera Fischer, S. Graf, H. P. Campos, Y. Z. German, M. D. Carmen Saldias and I. Alvarez, Cryobiology, 2006, 52, 17; (b) Y. Liu, J. Lu, H. Li, J. Wei and X. Li, Acta Biomater., 2015, 11, 114.

32 A. Hasan, A. Memic, N. Annabi, M. Hossain, A. Paul, M. R. Dokmeci, F. Dehghani and A. Khademhosseini, Acta Biomater., 2014, 10, 11.

33 W. M. Abbott, A. Callow, W. Moore, R. Rutherford, F. Veith and S. Weinberg, J. Vasc. Surg., 1993, 17, 746.

34 D. G. Seifu, A. Purnama, K. Mequanint and D. Mantovani, Nat. Rev. Cardiol., 2013, 10, 410.

35 J. T. Patterson, T. Gilliland, M. W. Maxfield, S. Church, Y. Naito, T. Shinoka and C. K. Breuer, Regener. Med., 2012, 7, 409. 